\title{
Intensive electrocaloric effect in the multilayer capacitor under equilibrium and nonequilibrium thermal conditions
}

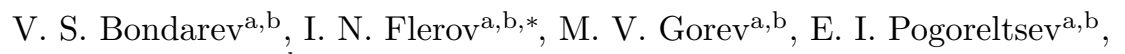 \\ M. S. Molokeeva,b, E. A. Mikhaleva ${ }^{a}$, A. V. Shabanov ${ }^{\mathrm{a}}$, A. V. Es'kov ${ }^{\mathrm{c}}$ \\ ${ }^{a}$ Kirensky Institute of Physics, Federal Research Center KSC SB RAS, 660036 \\ Krasnoyarsk, Russia \\ ${ }^{b}$ Siberian Federal University, 660074 Krasnoyarsk, Russia \\ ${ }^{c}$ School of Biotechnology and Cryogenic Systems, St. Petersburg National Research \\ University of Information Technologies, Mechanics, and Optics, St. Petersburg, 197101 \\ Russia
}

\begin{abstract}
Precise direct measurements of the intensive electrocaloric effect (ECE) in commercial multilayer capacitor based on doped $\mathrm{BaTiO}_{3}$ were performed using an adiabatic calorimeter. High reversibility of ECE studied under equilibrium thermal conditions was observed. The nonequilibrium thermal conditions caused by fixing the temperature of one of the ends of the linear EC element lead to the predominance of ECE when the electric field is turned off. The heat flow through the EC element appearing under the influence of a periodic electric field and depending on its frequency makes it possible to create a cooling cycle without thermal keys.
\end{abstract}

Keywords: Electrocaloric effect, Phase transition, Ferroelectrics

PACS: 77.70.+a; 64.70.K-; 65.40.-b

\footnotetext{
* Corresponding author

Email addresses: vbondarev@yandex.ru (V. S. Bondarev), flerov@iph.krasn.ru (I. N. Flerov), gorev@iph.krasn.ru (M. V. Gorev), pepel@iph.krasn.ru (E. I. Pogoreltsev), msmolokeev@mail.ru (M. S. Molokeev), katerina@iph.krasn.ru (E. A. Mikhaleva), alexch_syb@mail.ru (A. V. Shabanov), aeskow@gmail.com (A. V. Es'kov)
} 
Electrocaloric effect (ECE) in ferroelectric materials has a great potential in realizing solid-state cooling devices with compact size and high efficiency which are highly desirable for a wide range of applications such as on-chip cooling and temperature controlling for sensors, electronic devices, and medical instruments. ECE is associated with the reversible change in the temperature, $\Delta T_{A D}$, or entropy, $\Delta S_{E C E}$, under electric field variation under adiabatic and isothermal conditions, respectively. According to interrelations between the extensive and intensive ECE and polarization, $\Delta S_{E C E}=\int(\partial P / \partial T)_{E} d E$, $\Delta T_{A D}=-\left(T / C_{p}\right) \Delta S_{E C E}$, the largest values of both effects can be obtained near the ferroelectric phase transition point, where the derivative $(\partial P / \partial T)_{E}$ reaches the maximum magnitude [1]. Recent development in the design of the electrocaloric (EC) components and cooling systems has indicated that the intensive ECE equal to $\Delta T_{A D}=3 \mathrm{~K}$ would be enough to construct an EC solidstate refrigeration system with refrigeration costs comparable to todays vaporcompression systems [2]. Theoretical estimations have shown that in order to realize $\Delta T_{A D} \approx 6 \mathrm{~K}$ in $\mathrm{Ba}_{0.5} \mathrm{Sr}_{0.5} \mathrm{TiO}_{3}$, rather high electric field $E \approx 300 \mathrm{kV} / \mathrm{cm}$ is needed [3]. However, the breakdown field for bulk ferroelectric materials does not usually exceed $60 \mathrm{kV} / \mathrm{cm}$. On the other hand, thin ferroelectric films can support higher fields $E \approx 1000-2000 \mathrm{kV} / \mathrm{cm}$ which can be obtained at low voltage due to small thickness of films. This is the reason why gigantic intensive $\operatorname{ECE}\left(\Delta T_{A D} \approx 14 \mathrm{~K}\right)$ was observed in such kind of ferroelectric materials [4]. Unfortunately, thin films have a small thermal mass which brings about small EC heat. Multilayer ceramic capacitors (MLCCs) have been suggested [5, 6] as an alternative design for EC coolers since they combine high breakdown field due to rather thin layers $(<10 \mu \mathrm{m})$ as well as large thermal mass and as a result high cooling power. Investigations of ECE in the multilayer structures based on $\mathrm{BaTiO}_{3}$ have demonstrated $\Delta T_{A D} \leq 1 \mathrm{~K}$ at $E=300 \mathrm{kV} / \mathrm{cm}$ [5, 7, 8, 9, 10, 11, 12].

However, refrigerators with solid working body operating on the classic ECE associated with the applying/removal of a constant electric field to/from the ECE element have a serious disadvantage: heat switches are needed to provide thermal contact of solid refrigerant in turn with cooled object and environ- 
ment [11, 13, 14].

Recently, an original way was suggested to avoid such a serious problem [15, 16, 17, 18. The idea was to create a heat flux through the EC element. Theoretical modelling has shown that such an effect can be obtained realizing ECE under the periodic $E$ in the bulk EC element under nonequilibrium thermal conditions. The top of the linear element was thermally isolated and the bottom was kept at $T_{b o t}=$ const. As a result, in a certain temperature range, applying and removal of the electric field will be accompanied by the different values of $\Delta T_{A D}\left(|\Delta T|_{A D}^{O N}<|\Delta T|_{A D}^{O F F}\right)$. Periodic variations in the field, $E=0 \rightarrow E \neq 0 \rightarrow E=0$, will lead to fluctuations in the temperature of the top of the EC element, $T_{\text {top }}$. The gradual decrease of its average value down to $T_{\text {top }}<T_{b o t}$ will appear due to the nonequivalence of the heat fluxes when the field is turned on and off.

Experimental studies of ECE under nonequilibrium thermal conditions were performed on the multilayer capacitor based on $\mathrm{BaTiO}_{3}$ [11, [5] and the bulk triglycine sulphate crystal (TGS) [19, 20]. In the former case, in the temperature range investigated they have found $|\Delta T|_{A D}^{O N} \geq|\Delta T|_{A D}^{O F F}$ and as a result the heating of the sample under periodic $E$ [11. However, in the latter case, a decrease in the temperature $T_{t o p}$ compared to $T_{b o t}$ of the TGS element was detected in accordance with previous predictions [15, 18. Thus, despite a small difference $T_{\text {top }}-T_{\text {bot }}=-0.012 \mathrm{~K}$ at rather low $E=2.8 \mathrm{kV} / \mathrm{cm}$ a cooling effect was observed.

We assume that failure of the cooling observation in MLCC [11, 5] is due to at least two reasons. First, experiments were performed at high field $E=300 \mathrm{kV} / \mathrm{cm}$ which necessarily leads to the release of Joule heat. Second, to avoid the destruction of the capacitor under high voltage, the discharge process was carried out through an additional resistor which led to different rates of switching on/off the electric field, $(d E / d t)_{O F F}<(d E / d t)_{O N}$. Both reasons contribute to the observed interrelation $|\Delta T|_{A D}^{O N}>|\Delta T|_{A D}^{O F F}$. At the same time, measurements of the ECE in TGS were carried out at equal rates of switching on/off the electric field [19, 20]. 
In the present paper, we performed precise direct measurements of the intensive ECE in multilayer capacitor based on $\mathrm{BaTiO}_{3}$ (MLCBT) by means of a homemade adiabatic calorimeter which is characterized by very high sensitivity to the small temperature change [19, 20, 21]. A commercially available MLCBT $(100 \mu \mathrm{F})$ consisting of 200 interdigitated layers of $\mathrm{BaTiO}_{3}$ and $\mathrm{Ni}$ electrodes was used. The nominal thickness of the layers is $6.5 \mu \mathrm{m}$ for $\mathrm{BaTiO}_{3}$ and $2.0 \mu \mathrm{m}$ for Ni (Fig. 1(a)). To obtain correct information on ECE and the temperature gradient $\Delta T=T_{t o p}-T_{b o t}$, the study was carried out with the equal values of $(d E / d t)_{O F F}$ and $(d E / d t)_{O N}$ under voltage of $10 \mathrm{~V}$, i.e. $E=15.4 \mathrm{kV} / \mathrm{cm}$, which is close to $15 \mathrm{kV} / \mathrm{cm}$ recommended by the manufacturer in order to avoid a deterioration in the functional properties of the capacitor.

The structural morphology of the cut perpendicular to the $\mathrm{BaTiO}_{3}$ and Ni layers was examined using a scanning electron microscope (SEM) Hitachi TM3000 (Hitachi High - Technologies Co., Ltd., Tokyo, Japan) and is presented in Fig. 1(b). The SEM image clearly demonstrates that the surface consists of alternating layers of ferroelectric (light broad stripes) and metal (dark narrow stripes) components contacting closely each other.

(a)
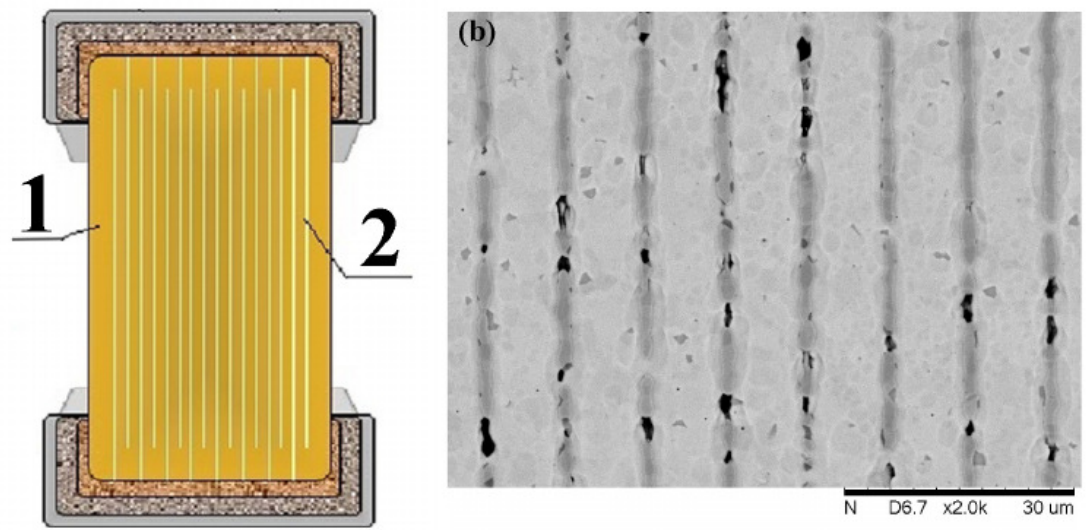

Figure 1: (a) MLCBT cross-sectional schematic (1 - doped $\mathrm{BaTiO}_{3} ; 2$ - Ni electrodes). (b) SEM image of the cut perpendicular to the $\mathrm{BaTiO}_{3}$ and $\mathrm{Ni}$ layers.

The X-ray powder diffraction data were collected at room temperature with 
a PANalytical X'Pert PRO diffractometer equipped with a PIXcel solid state detector and a secondary graphite monochromator $(\mathrm{Cu}-\mathrm{K} \alpha$ radiation). Rietveld refinement shows the presence of $\mathrm{Ni}$ and $\mathrm{BaTiO}_{3}$ doped with $\mathrm{Sn}^{4+}$ which gives the following chemical formula: $\mathrm{BaTi}_{0.86} \mathrm{Sn}_{0.14} \mathrm{O}_{3}$ (BTSO).

Figure 2(a) represents the behavior of the permittivity measured at $1 \mathrm{kHz}$ using an E7-20 immittance meter. One broad smeared peak with a maximum at about $302 \mathrm{~K}$ was found which is characteristic for relaxors and coincides with manufacturers information about anomalous behavior of $\varepsilon$ of MLCBT in wide temperature range [22].

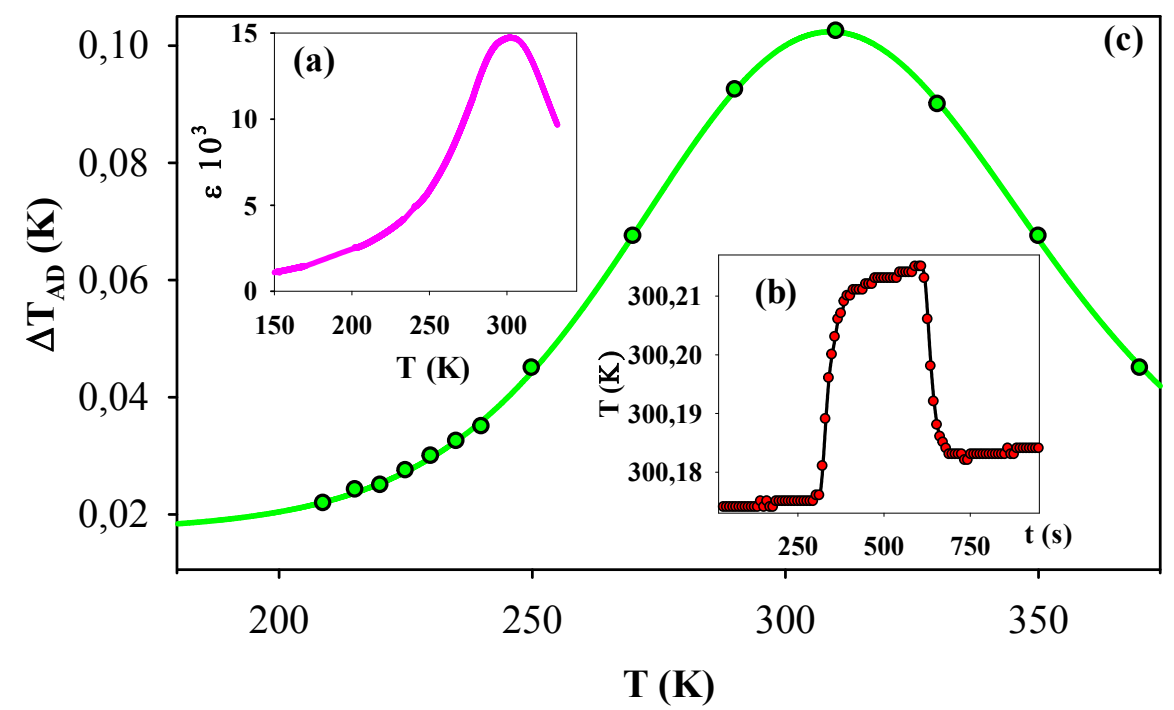

Figure 2: (a) Temperature dependence of the permittivity $\varepsilon$ measured at frequency of $f=1 \mathrm{kHz}$. (b) The time dependence of the temperature of the MLCBT + heater system under successive electric field variation at $S=$ const: $E=0 \rightarrow E=15.4 \mathrm{kV} / \mathrm{cm} \rightarrow E=0$. (c) Temperature dependence of the intensive ECE at $E=15.4 \mathrm{kV} / \mathrm{cm}$ recalculated for pure dielectric medium BTSO.

Direct measurements of the intensive ECE were performed on the MLCBT + heater system by means of adiabatic calorimeter at the pressure of about $10^{-5} \mathrm{~mm} \mathrm{Hg}$. A dc power homemade supply was used to apply the electric field 
on the sample. To minimize the thermal losses, contact wires with small diameters $\sim 0.05 \mathrm{~mm}$ were used. To obtain information on $\Delta T_{A D}$ only in MLCBT, the heat capacity of the heater was measured in a separate experiment. Since the real masses of Ni-electrodes, plastic coating, etc, are unknown, the values $\Delta T_{A D}$ relating to BTSO were obtained using the coefficient $\triangle T_{A D}^{B T S O} / \triangle T_{A D}^{M L C B T}=1.47$ evaluated for the similar capacitor [5].
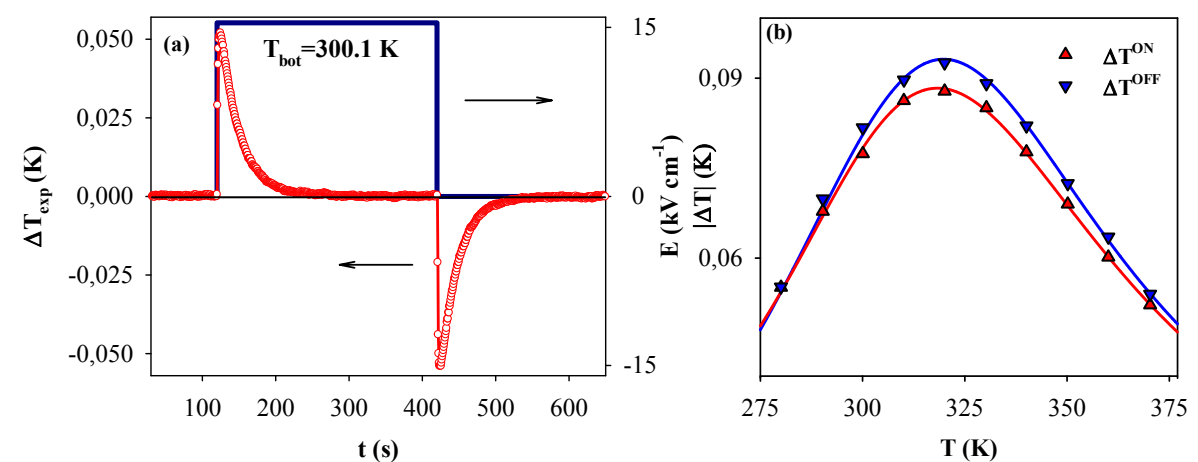

Figure 3: (a) The profile of electric field variation $E(t)$ and associated intensive EC response, $\Delta T_{\text {exp }}(t)=T_{t o p}-T_{b o t}$, at $T_{b o t}=300.1 \mathrm{~K}$. (b) Temperature dependences of the intensive ECE in applying $|\Delta T|_{O N}$ and removal $|\Delta T|_{O F F}$ modes at $E=15.4 \mathrm{kV} / \mathrm{cm}$.

Experiments were carried out under two different thermal conditions used by us studying TGS [20]: 1) at $S=$ const under applying/removal of an electric field with subsequent exposure $E=$ const for 5 - 10 min.; 2) the same procedure under nonequilibrium thermal conditions at $T_{b o t}=$ const. Hereinafter, these variants will be labelled as Var 1 and Var 2, respectively.

In Var 1, the temperature drift of the MLCBT + heater system at different temperatures was chosen within $d T / d t \approx \pm(1-5) 10^{-4} \mathrm{~K} / \mathrm{min}$. Applying and removal of a constant electric field to/from electrodes of MLCBT resulted in a rapid increase/decrease in temperature of the system due to ECE in BTSO (Fig. 2(b)). It is also seen that the rate of the temperature change, $d T / d t$, in the process of $E=$ const is greater than before the applying of the electric field and becomes equal to it after the field is switched off. This experimental fact 
unequivocally indicates the release of Joule heat in the BTSO component even under a relatively low electric field, $E=15.4 \mathrm{kV} / \mathrm{cm}$, compared to $E=300 \mathrm{kV} / \mathrm{cm}$ used in the previous studies [11, [5]. The corresponding additional increase in the temperature of the MLCBT + heater system under the field was about 10\%. Figure 2(c) impacts the temperature dependence of the intensive ECE in MLCBT at $E=15.4 \mathrm{kV} / \mathrm{cm}$ which is also demonstrates only one broad peak similar to $\varepsilon(T)$ (Fig. 2(a)). The maximum of $\Delta T_{A D}=0.104 \mathrm{~K}$ was found at $T_{\max }=309 \mathrm{~K}$. which is higher than the temperature of $\varepsilon_{\max }$. This phenomenon is associated with rather large coefficient $d T_{\max } / d E \approx 0.5 \mathrm{Kcm} / \mathrm{kV}$. Due to the adiabatic conditions of the ECE measurements the error in determining the value $\Delta T_{A D}$ was less than $\pm 2 \cdot 10^{-4} \mathrm{~K}$ The reversibility of ECE was also rather high. The difference between the magnitudes of $\Delta T_{A D}$ realized in the regimes of applying and removal of $E$ did not exceed the uncertainty above.

To realize Var 2, the bottom of MLCBT was glued to the copper holder with $T_{b o t}=$ const $\pm 10^{-4} \mathrm{~K}$ mounted on an adiabatic screen. When an electric field is switched on/off, $T_{\text {top }}$ rapidly increases/decreases due to the ECE and then, at $E=$ const, relaxed gradually to $T_{b o t}, \Delta T_{\exp } \rightarrow 0$ (Fig. 3(a)). Figure3(b) depicts the time dependence of $\Delta T$ recalculated for the pure BSTO as it was done above for $\Delta T_{A D}^{B T S O}$. Two interesting features were observed. First, the irreversibility of the intensive ECE, $|\Delta T|^{O F F}>|\Delta T|^{O N}$, was observed in a temperatures range of 300-370 K. Second, the maximum EC response $|\Delta T|^{O F F}$ under nonequilibrium thermal conditions is lower by about $10 \%$ compared to $\Delta T_{A D}^{\max }$ observed in Var 1 at $S=$ const at the same temperature $T_{\max }=320 \mathrm{~K}$ (Fig. 2(c)). This can be due to the occurrence of a heat flux in the EC element during the process $(E=0) \rightarrow(E=15.4 \mathrm{kV} / \mathrm{cm})$. The maximum difference $|\Delta T|^{O F F}-|\Delta T|^{O N}=0.005 \mathrm{~K}$ was observed also at $320 \mathrm{~K}$. The inequality of the ECE values under applying/removal $E$ means that the amounts of the released and absorbed heat are not equal to each other. The temperature gradient $\Delta T=T_{t o p}-T_{b o t}<0$ occurring on the sample leads to the heat flow directed from the top to the bottom of MLCBT.

Taking into account rather large value $d T_{\max } / d E, T_{b o t}=310 \mathrm{~K}$ was chosen for 
further measurements of ECE under nonequilibrium thermal conditions under periodic electric field. Figure 4(a) shows that the steady thermal state of the system was achieved after several pulses of $E(f=0.025 \mathrm{~Hz})$. The temperature $T_{\text {top }}$ of the EC element oscillates around some average value $T_{b o t}-\left[\left(\Delta T^{O N}+\right.\right.$ $\left.\left.\Delta T^{O F F}\right) / 2\right]$.
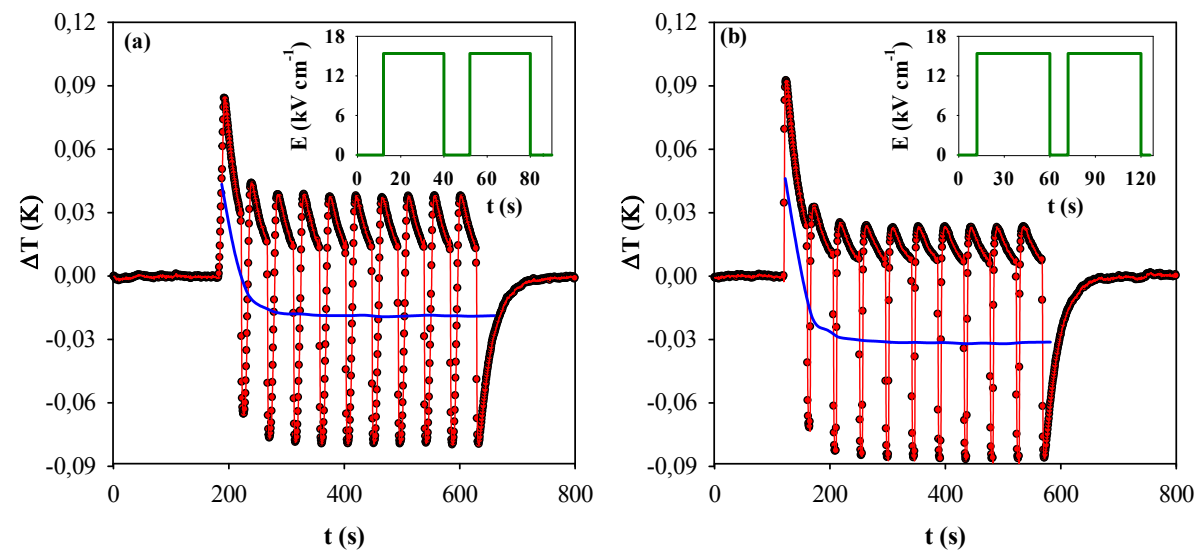

Figure 4: The time dependences of the temperature difference $\Delta T$ under a periodic electric field of $E=15.4 \mathrm{kV} / \mathrm{cm}$ of different frequencies at $T_{\text {bot }}=310 \mathrm{~K}$ : (a) $f=0.025 \mathrm{~Hz}$, (b) $f=0.016 \mathrm{~Hz}$. Solid lines show behavior of the average $\Delta T$ values. Insets depict the profile of the electric field.

The decrease in the $E$ frequency is accompanied by an increase in the cooling effect. The value $\left[\left(\Delta T^{O N}+\Delta T^{O F F}\right) / 2\right]^{\max }=-0.032 \mathrm{~K}$ reached at $f=0.016 \mathrm{~Hz}$ exceeds $-0.019 \mathrm{~K}$ at $0.025 \mathrm{~Hz}$ (Fig. 4(a) and 4(b)). Of course, the observed effect of cooling is not as large as we would like, but it is larger the value found in TGS [19, 20. We can confidently assume that an increase in the electric field strength will also lead to an increase in the temperature gradient accompanied by an increase of the heat flow through the EC element. However, the resistance of dielectrics used in the capacitor should be very high to avoid or at least reduce the release of Joule heat.

In conclusion, EC properties of commercially available multilayer capacitor based on doped $\mathrm{BaTiO}_{3}$ were studied under the electric field of $E=15.4 \mathrm{kV} / \mathrm{cm}$ 
using an adiabatic calorimeter. A rather good agreement between the maximum values of the intensive ECE induced under the equilibrium ( $d S=$ const) and nonequilibrium $\left(T_{b o t}=\right.$ const $)$ thermal conditions was found. In the latter case, an inequality between the temperature increase and decrease under applying and removal of $E,|\Delta T|^{O N}<|\Delta T|^{O F F}$ was observed. It was shown that this effect under the periodic electric field allows one to realize a heat flow through the EC element and as a result to exclude the thermal switches from the refrigeration process. One of the ways to increase the cooling capacity is associated with the use of multilayer structures based on materials with very low electrical conductivity, which allows the application of fields with a higher strength.

\section{Acknowledgements}

The reported study was funded by Russian Foundation for Basic Research, Government of Krasnoyarsk Territory, Krasnoyarsk Region Science and Technology Support Fund to the research, project no. 16-42-240428 p-a.

\section{References}

[1] M. Valant, Prog. Mater. Sci. 57 (2012) 980-1009.

[2] Y. V. Sinyavski, G. E. Lugansky, N. D. Pashov, Cryogenics 32 (1992) 28-31.

[3] I. Ponomareva, S. Lisenkov, Phys. Rev. Lett. 108 (2012) 167604.

[4] A. S. Mischenko, Q. M. Zhang, J. F. Scott, R. W. Whatmore, N. D. Mathur, Science 311 (2006) 270.

[5] S. Kar-Narayan, N. D. Mathur, J. Phys. D: Appl. Phys. 43 (2010) 032002.

[6] N. A. S. Smith, M. K. Rokosz, T. M. Correia, J. Appl. Phys. 116 (2014) 044511 .

[7] S. Kar-Narayan, S. Crossley, X. Moya, V. Kovacova, J. Abergel, A. Bontempi, N. Baier, E. Defay, N. D. Mathur, Appl. Phys. Lett. 102 (2013) 032903. 
[8] B. Kwon, I. J. Roh, S. H. Baek, S. K. Kim, J. S. Kim, C. Y. Kang, Appl. Phys. Lett. 104 (2014) 213902.

[9] M. Quintero, L. Ghivelder, F. Gomez-Marlasca, F. Parisi, Appl. Phys. Lett. 99 (2011) 232908.

[10] M. Quintero, P. Gaztañaga, I. Irurzun, Appl. Phys. Lett. 107 (2015) 151901.

[11] R. I. Epstein, K. J. Malloy, J. Appl. Phys. 106 (2009) 064509.

[12] S. Kar-Narayan, N. D. Mathur, Appl. Phys. Lett. 95 (2009) 242903.

[13] Y. D. Wang, S. J. Smullin, M. J. Sheridan, Q. Wang, C. Eldershaw, D. E. Schwartz, Applied Physics Letters 107 (2015) 134103.

[14] S. J. Smullin, Y. Wang, D. E. Schwartz, Appl. Phys. Lett. 107 (2015) 093903.

[15] S. F. Karmanenko, O. V. Pakhomov, A. M. Prudan, A. S. Starkov, A. Eskov, J. Eur. Ceram. Soc. 27 (2007) 3109-3112.

[16] A. Khodayari, S. Mohammadi, IEEE Trans. Ultrason. Ferroelectr. Freq. Contr. 58 (2011) 503-507.

[17] Y. Bai, G.-P. Zheng, S.-Q. Shi, J. Appl. Phys. 108 (2010) 104102.

[18] A. V. Es'kov, S. F. Karmanenko, O. V. Pakhomov, A. S. Starkov, Phys. Solid State 51 (2009) 1574-1577.

[19] V. S. Bondarev, E. A. Mikhaleva, M. V. Gorev, I. N. Flerov, Phys. Status Solidi B 253 (2016) 2073-2078.

[20] V. S. Bondarev, E. A. Mikhaleva, I. N. Flerov, M. Gorev, Phys. Solid State 59 (2017) 1118-1126.

[21] A. V. Kartashev, I. N. Flerov, N. V. Volkov, K. A. Sablina, Phys. Solid State 50 (2008) 2115-2120.

[22] M. D. Waugh, Electronic Engineering Times Europe August (2010) 34-36. 